\title{
Sobre la justificación de las proposiciones ante los desafíos escépticos
}

\section{How to justify propositions when facing skeptical challenges}

Federico Matías Pailos

(Universidad de Buenos Aires, CONICET)

Recibido: 26/08/2013

Aceptado: 14/11/2013

\section{Resumen}

Los desafíos escépticos cuestionan la justificación de las proposiciones que aceptamos. Pero es posible justificar en términos probabilísticos cada una de las proposiciones empíricas aceptadas. Para eso, su probabilidad condicional al resto de las proposiciones aceptadas, deberá ser mayor que su probabilidad absoluta. Esta justificación es circular, pero virtuosa. Sin embargo, carece de eficacia dialéctica frente al escéptico.

Palabras clave: Justificación, escepticismo, probabilidad, creencia, proposición, circularidad, certeza, aceptación.

\section{Abstract}

Skeptical challenges question that the propositions one accepts are justified. But it is possible to justify each one of them in probabilistic terms. In order to do that, their probability, conditional to the rest of the accepted propositions, must be higher than their absolute probability. This justification is circular, but virtuous. Nevertheless, it has no dialectical efficiency against the skeptic.

Keywords: Justification, skepticism, probability, belief, proposition, circularity, certainty, acceptance. 
El escepticismo divide aguas. Muchas, y de varias maneras. Un modo de interpretar lo que hay en juego con los desafíos escépticos, es pensar que el debate de fondo se da entre escépticos y dogmáticos. Los primeros niegan o ponen en duda que podamos tener justificación o conocimiento (en general, o acerca de un ámbito particular). Los últimos afirman que la justificación o el conocimiento en cuestión se derraman desde unas cuantas proposiciones o creencias básicas, que están justificadas o son conocidas automáticamente, o que no necesitan justificación. Eventualmente, una opción a estas posiciones es la salida infinitista, que afirma que tenemos justificación o conocimiento de las proposiciones puestas en duda por los escépticos, porque hay una cadena infinita de justificaciones en la que la justificación en cuestión descansa. El infinitismo, no obstante, dista de ser la posición mayoritaria. ${ }^{1}$ No obstante, es una posición más en el debate acerca de la justificación de algunas proposiciones centrales, como que hay un mundo o que nuestros sentidos son fiables. Este trabajo se ocupará de ese tema.

Pero el enfoque tradicional sobre el tema no es el único posible. Otro modo de enfocar la cuestión del escepticismo es pensar que la división básica no es entre escépticos y dogmáticos (o infinitistas, eventualmente), sino entre dos paradigmas más abarcativos: el modelo cartesiano de duda/creencia, y el modelo a veces llamado "pragmatista" de creencia/duda. El cartesiano hace hincapié en que no debemos mantener ninguna creencia o aceptar ninguna proposición -porque ninguna creencia o proposición está justificada por defecto- a menos que cuente con una razón positiva que la avale. El pragmatista, interesado básicamente en las normas que gobiernan, que es racional que gobiernen, o que deberían gobernar si fuéramos racionales, el cambio de creencias, sostiene que no debemos poner en duda ninguna creencia -o "proposición aceptada" -2 a menos que tengamos una razón positiva para hacerlo. ${ }^{3}$ Los cartesianos, en suma, ponen la carga de la prueba en la creencia, mientras que los pragmatistas lo hacen en la duda. ${ }^{4}$

\footnotetext{
1 Para una defensa del infinitismo, ver Klein, K.: "Why not Infinitism?", en Cobb-Stevens, R. (ed.), Epistemology: Proceedings of the Twentieth World Congress in Philosophy, 5, 2000, pp. 199-208, y Klein, K.: "Infinistism is the Solution to the Regress Problem", en Sosa, E. y Steup, M. (eds.), Contemporary Debates in Epistemology, 2005, pp. 131-139. Más adelante, ahondaré en lo que parece estar mal con el infinitismo.

2 Como no es nada claro que sea correcto hablar de normas que gobiernen el cambio de creencias -porque no es nada claro que el cambio de creencias sea o pueda ser voluntario-, optaré, en general, por hablar de las normas que gobiernan la aceptación o el cambio de proposiciones que se acepta.

3 Algunas de las posiciones que en este trabajo llamo "pragmatistas" son defendidas en Jeffrey, R.: The Logic of Decision. New York: McGraw Hill, 1966. Levi, I.: "On Indeterminate Probabilities", Journal of Philosophy 71: 391-418, 1974. Levi, I.: The Enterprise of Knowledge. Cambridge, Mass: The MIT Press, 1980.

4 Esta diferenciación no se solapa con la primera dado que se puede ser un dogmático cartesiano (y creer que de hecho se cuentan con buenas razones que justifican las creencias de las que el escéptico duda), así como se puede ser un escéptico pragmatista (porque se crea que se tienen buenas razones para dudar de esta o aquella creencia básica).
} 
Para un defensor del modelo creencia/duda, no es que, como defienden los cartesianos dogmáticos, haya proposiciones que no necesiten justificación -i.e., las proposiciones llamadas "básicas"-, sino que la mera formulación de alternativas escépticas no constituyen, por sí mismas, buenas razones para dudar de ellas. Esto puede llevar a cuestionarnos la conveniencia de aceptar o mantener proposiciones que no se cree justificadas - porque lo que requiere justificación es, en primera instancia, las dudas, y no las creencias, o las proposiciones que se acepta-. Acaso tal incomodidad mengue si se abraza la idea de que la justificación no es un atributo de proposiciones, afirmaciones o creencias, sino de los cambios de creencias (o de los cambios en el conjunto de proposiciones aceptadas). Atribuir justificación a las proposiciones aceptadas o creídas sería, así, un error categorial. Pero de todas formas, subsiste la impresión de que no es extraño, ni mucho menos, atribuir justificación a proposiciones, afirmaciones o creencias. Es decir, tal posición contraría un uso habitual de la idea de justificación. Pero hay, en esta incomodidad, algo más allá que la resistencia a un eventual proyecto de reforma lingüística (pragmatista), porque parece sensato suponer que las proposiciones que se aceptan -las creencias que se tienen- comparten, o deberían compartir, si fuera racional mantenerlas, algún tipo de virtud epistémica. Tienen que ser "buenas", desde el punto de vista epistémico. La posición pragmatista, tal como fue presentada, no dice nada con respecto a este punto, así que, al menos, es menos completa que lo deseable. Además, si quisiéramos dar un nombre a esa "bondad” epistémica, ¿no sería "justificación” un término suficientemente adecuado? Es decir, ante la pregunta de qué tiene esa proposición particular para incorporarla o mantenerla en el conjunto de proposiciones aceptadas, las respuestas "hay buenas razones para pensar que es verdadera" o "está justificada" parecen atinadas. Un pragmatista podría insistir con que la pregunta correcta acerca de las proposiciones aceptadas no sería “¿qué las justifica?”, sino “¿hay razones para abandonarla?". De todas formas, no solo suena extraño negar que puedan estar justificadas, sino también negar que estén justificadas. La pregunta que explica esa extrañeza es: ¿por qué somos racionales en mantenerlas $-\mathrm{y}$, originalmente, por qué fuimos racionales en aceptarlas-, si no contamos con una buena justificación a su favor? Si una proposición forma parte del conjunto en cuestión, debe haber buenas razones "que hablen a su favor". ¿Por qué? Porque ellas son las que explican que seamos racionales al aceptar o mantener la proposición.

Entre esas creencias -o entre el conjunto de proposiciones aceptadas- suelen estar -0 , al menos, parece sensato decir que deberían estar- estas dos:

1- Hay un mundo.

2- Nuestro sistema perceptivo es confiable. 56

\footnotetext{
5 De hecho, para lo que sigue, con (2) va a alcanzar.

6 Un mecanismo de producción de afirmaciones, juicios, proposiciones o creencias es confiable, cuando el resultado de su operación está constituido por portadores de valor de verdad (afirmaciones, jui-
} 
Pensemos ahora en el asunto de las razones a favor de una proposición que es parte del conjunto de proposiciones aceptadas. Un defensor del modelo creencia/duda debería tratar de decir por qué es racional aceptarlas (o mantenerlas). Por ejemplo, podría afirmar que esas proposiciones de hecho están justificadas, y lo están, por caso, porque puede justificarse que $p$, dado que veo que $p$ (donde $p$ es una proposición perceptual). Esta justificación puede entenderse en términos probabilísticos: $\mathrm{P}(p /$ veo que $p)>\mathrm{P}(p)$, y además, es mayor que la probabilidad condicional de las alternativas escépticas. 78 En particular, la justificación de que hay una mano puede darse porque $\mathrm{P}$ (hay una mano/tengo la experiencia visual de que hay una mano $)>\mathrm{P}($ hay una mano $)$.

Entonces parece que (A) si se mantiene racionalmente una proposición, debe haber una buena razón que la avale, pero (B) tampoco parece ser suficiente con plantear una duda escéptica - por ejemplo, a través de una alternativa escépticapara ponerla en duda. Esa alternativa escéptica debe ser, al menos, tan probable como la proposición puesta en cuestión, dado el resto de las proposiciones aceptadas. Es decir, $\mathrm{P}(p /$ resto de las proposiciones aceptadas) $>\mathrm{P}$ (una alternativa escéptica a $p$ /resto de las proposiciones aceptadas). Más aún: si de hecho es racional que la proposición en cuestión sea parte del conjunto mentado, entonces debe haber -i.e., sería mejor que hubiera-, en algún sentido, razones a su favor. Si bien no es el único tipo de razones a favor que puede haber, muchas veces ellas serán de este estilo: la probabilidad de tal proposición, dada la verdad de alguna otra proposición aceptada, es mayor a la probabilidad no condicionada de esa proposición. ${ }^{9}$

Surge aquí la siguiente objeción a este planteo: la justificación de las proposiciones aceptadas es circular, y por tanto, viciosa. Es cierto que la justificación propuesta es circular. Lo es, porque podría -y debería- justificarse cada proposición que integra el conjunto de proposiciones aceptadas de esta manera (es decir, en virtud de su probabilidad condicional al resto de las proposiciones, que a su vez se jus-

cios, proposiciones o creencias) que, en una proporción sustantiva de los casos (al menos mayor al $50 \%$, pero parece razonable pedir mucho más que esto), son verdaderos.

7 Esto vale, en principio, solo para proposiciones empíricas. En particular, no es sencillo pensar en una $p$ que sea una tautología, tal que $\mathrm{P}(p /$ resto de las proposiciones aceptadas $)>\mathrm{P}(p)$. Y si se acepta que la probabilidad absoluta de las tautologías es 1, podemos concluir que seguramente no va a pasar, porque no puede haber un grado de probabilidad superior al máximo.

8 Esto, de hecho, parece lo principal. En teoría, esto no es incompatible con que pueda haber una $p$ tal que $\mathrm{P}(p /$ resto de las proposiciones aceptadas $)>\mathrm{P}$ (una alternativa escéptica a $p /$ resto de las proposiciones aceptadas $)$-y que esto valga para cada alternativa escéptica a $p$-, pero $\mathrm{P}(p)>\mathrm{P}(p /$ resto de las proposiciones aceptadas).

9 Otras razones a favor de la proposición en cuestión pueden ser que esa proposición se deduce del conjunto de creencias aceptadas, o que es la mejor explicación de un cierto fenómeno, o que existe un consenso a su favor dentro de la comunidad relevante de expertos. De todas formas, no es seguro que todas estas razones no puedan explicarse en los términos probabilísticos planteados. En cualquier caso, nada de lo que se dice a continuación depende de este punto. 
tifican, en parte, en su probabilidad condicional a un conjunto de proposiciones del que la proposición inicialmente justificada también forma parte). La probabilidad condicional de cada una de estas proposiciones debería ser mayor que la probabilidad condicional de cada una de sus alternativas escépticas (con respecto al resto de las proposiciones aceptadas). Cierto: esta justificación es circular. Para salvar la crítica, debería defenderse que no solo no es viciosamente tal, sino que es virtuosa. Un modo -abductivo- de hacer esto es sostener que este tipo de justificación nos permite rescatar un número mayor de las proposiciones que aceptamos (o de las creencias que tenemos) que el resto de los modos de justificación. Hay, además, casos claros de justificación viciosa, de los que esta justificación se distingue. Podría decirse lo siguiente: cuando dos o más elementos que pertenecen al conjunto de proposiciones aceptadas se apoyan inferencialmente entre sí, estamos frente a un caso de justificación virtuosa. Cuando ninguno de estos elementos pertenece al conjunto de proposiciones aceptadas, la justificación circular que pudiera haber entre ellos es viciosa. Es decir, las justificaciones virtuosas apelan a elementos que forman parte del conjunto de proposiciones aceptadas. Las viciosas, no.

De este modo, a su vez, logramos que sea posible que todas las proposiciones que aceptamos estén justificadas, y evitamos, a su vez, caer en posiciones infinitistas. ¿Cuál es el problema del infinitismo, entendido como una posición diferente al tipo de justificación circular defendido en este trabajo? ${ }^{10}$ Que es difícil pensar que haya una cadena infinita de proposiciones aceptadas, tales que cada una justifique a la anterior, sin que ninguna de esas proposiciones se repita -porque de lo contrario caería en la circularidad-, tal que cada elemento que pretende justificar de hecho lo haga, es decir, que aumente la probabilidad de lo que justifica. En particular, si se empieza con algo tan básico como que hay un mundo. Acaso esta proposición pueda justificarse en que nuestros sentidos son confiables. Pero esta proposición, ¿en qué puede justificarse? ¿En que es la mejor explicación de la propia supervivencia? ¿En que es la mejor explicación del propio éxito evolutivo? Pero, ¿no suponen ambas cosas que hay un mundo? Nuevamente, la justificación es circular. ( $\mathrm{Si}$ justificamos una proposición con un conjunto de proposiciones, los resultados seguramente van a ser los mismos en el caso de que queramos justificar la proposición aceptada de que hay un mundo.) La alternativa es justificarlas en proposiciones que no conlleven circularidad en la justificación. Pero entonces probablemente lo que se resienta sea el vínculo de justificación entre las proposiciones. En particular, acaso se pueda justificar la proposición de que nuestro sistema perceptual es confiable, con la proposición de que tengo la fuerte impresión de que esto es así. (Y esta, a su

\footnotetext{
$10 \mathrm{Si}$ acepta la circularidad, entonces no se entiende cuál sea su ventaja (ni su diferencia sustancial) frente a la posición que se defiende en este trabajo. El enfoque que se defiende en este trabajo, por su parte, no se compromete con la existencia de una cadena infinita de justificaciones. (Aunque sí con infinitas justificaciones, pero solo si el conjunto de proposiciones aceptadas es infinito.)
} 
vez, con la fuerte impresión de que esto es así, y proceder de este modo al infinito.) Pero no es nada claro en qué sentido $\mathrm{P}$ (hay un mundo/tengo la fuerte impresión de que hay un mundo) $>\mathrm{P}$ (hay un mundo). (De hecho, más bien parece que $\mathrm{P}$ (hay un mundo $)>\mathrm{P}$ (hay un mundo/tengo la fuerte impresión de que hay un mundo).) Esta es la principal sospecha acerca de lo adecuado de la salida infinitista: que cada eslabón de la cadena aumente la probabilidad del eslabón anterior. ${ }^{11}$

Esta interpretación probabilística de la justificación de proposiciones aceptadas no es dogmática -como sí lo es, por ejemplo, la defendida por Pryor, ${ }^{12}$ dado que, en principio, permite $-\mathrm{y}$ exige- que cualquier proposición que sea parte del conjunto de proposiciones aceptadas -conjunto al que presuntamente también pertenecen las proposiciones "básicas" del dogmático-, reciba justificación. ${ }^{13}$ Porque, de modo análogo, se podría pensar que la creencia de que hay un mundo puede recibir apoyo de varios lados; por ejemplo, de ser la mejor explicación -o, al menos, una suficientemente buena- del conjunto en general coherente de las propias percepciones, y de que estas percepciones se puedan, en algunos casos, prever. ${ }^{14}$ Pero además, puede afirmarse que estas proposiciones no solo son intuitivas, sino que también son razonables ${ }^{15}$ y compartidas por la amplia mayoría de los individuos y, en particular, por la mayoría de los filósofos. 16

Más aún: puede afirmarse que estas proposiciones están justificadas por el método filosófico correcto, que, según el propio Pryor, sería el siguiente: "we start with what it seems intuitively natural to say about perception, and we retain that

11 Para otra crítica -dogmática- al infinitismo, véase Turri, J.: "On the Regress Argument for Infinitism", Synthese 166:1, 2009, pp. 157-163.

12 En Pryor, J.: "The Skeptic and the Dogmatist", Nous, 4, 2000, pp. 517-549. Pryor, J.: "What' s Wrong with Moore' s Argument?", en Sosa, E. y Villanueva, E. (eds.) Philosophical Issues, 14, Epistemology, Atascadero: Blackwell Publishing. pp. 349-378, 2004.

13 Pero debe notarse, de todas formas, que esto no confronta en ningún otro sentido con la idea de la justificación de las proposiciones preceptúales de Pryor, quien acepta que la justificación es derrotable y que es inmediata, en el sentido de que "inmediato" solo excluye que la justificación provenga de otras creencias, porque esto no excluye que venga de otras proposiciones. Por ejemplo, que $\mathrm{P}(p /$ veo que $p)>\mathrm{P}(p)$. (O de que "veo que p", si se cree que cosas como " $\mathrm{P}(p /$ veo que $p)>\mathrm{P}(p)$ " no son, estrictamente, proposiciones.)

14 Esto también podría entenderse en términos probabilísticos.

15 Creer que mis sentidos son confiables, de hecho, parece una mejor explicación de la propia experiencia fenoménica que postular un perpetuo estado alucinatorio, fruto del obrar de un genio maligno o de las malas artes de una cubeta de última generación. Así, puede entenderse que es más razonable porque su probabilidad condicional al resto de las proposiciones aceptadas es mayor que su probabilidad absoluta y, a la vez, mayor -o, al menos, tan grande como- el resto de las hipótesis competidoras. 16 Parece razonable postular que el consenso aumenta la probabilidad de la verdad de una proposición. En general, cuánto más individuos relevantes crean que $p$, más probable es que $p$ sea verdadera. Desde ya, el número no es el único ni el principal factor a la hora de determinar la probabilidad de una proposición. 
natural view until we find objections that require us to abandon it". ${ }^{17}$ Sea o no el único método filosófico correcto, sí es ampliamente utilizado por buena parte de los filósofos. Y es un método correcto, al menos, si las intuiciones en cuestión no son las de los legos (las de cualquier hablante competente), sino las de los expertos. Ciertamente, esta metodología ha sido desafiada, en particular por los partidarios de la filosofía experimental. ${ }^{18}$ Pero no le faltan defensores. El principal de ellos, probablemente, sea Williamson, ${ }^{19}$ quien, no obstante, es renuente a hablar de intuiciones. Lo que sostiene Williamson es que, con respecto a un área en cuestión, debemos confiar más en los juicios de los expertos que en el de los legos. Si la discusión es acerca de física sub-atomómica, debemos confiar más en el juicio de los físicos; si es acerca de topología, debemos confiar más en el de los topólogos. Y si la discusión es acerca de filosofía, los juicios relevantes son los de los expertos. En efecto, sus juicios -sus intuiciones, que son un tipo de juicios- son falibles. Nadie lo niega. Pero el punto es que son confiables, y en una medida mucho mayor que los de los legos en la materia. ${ }^{20}$

El punto es el siguiente: es cierto que los expertos saben más que los legos sobre el tópico del que son expertos. Pero todavía no queda claro por qué del hecho de que las teorías de los expertos sean mejores que las del lego, se sigue que sus intuiciones sobre el punto también lo van a ser. Una explicación posible de este punto es la que ofrece James McBain. ${ }^{21}$ La idea es la siguiente: no hay intuiciones puras. Cuando un individuo afirma que un caso dado de creencia verdadera y justificada es un caso de conocimiento -en un caso Gettier-, su juicio está informado por sus teorías informales ["folk"] acerca del conocimiento, la creencia, la justificación y la verdad. Toda teoría que el individuo suscriba al respecto, o a propósito de asuntos relaciones, influye en el modo en que creerá correcto aplicar conceptos a situaciones particulares, o relacionarlo con otros conceptos. Pero toda teoría influye, y no solo las que suscribe informalmente. El experto tendrá mejores teorías que el lego, las que también influirán en su juicio. Pero además, el experto habrá pensado más

17 En Pryor, J.: "The Skeptic and the Dogmatist", Nous, 4, 2000, pp. 517-549, p. 538.

18 Las peculiaridades de este ataque pueden verse, por ejemplo, en Machery, E., Mallon, R., Nichols, S. y Stich, S.: "Semantics Cross-Cultural Style" Cognition 92: B1-B12, 2004, y Weinberg, J., Nichols, S. y Stich, S.: "Normativity and Epistemic Intuitions" Philosophical Topics, 29: 2001, pp. 429-460.

19 En Williamson, T.: "Philosophical 'Intuitions' and Scepticism about Judgement", Dialectica Vol. 58, No 1, 2004, pp. 109-153. Williamson, T.: "Philosophical Expertise and the Burden of the Proof", en http://www.philosophy.ox.ac.uk/_data/assets/pdf_file/0013/19300/metaphilosophy.pdf(4 de Enero de 2013).

20 Un punto análogo puede hacerse con respecto a las inclinaciones a emitir juicios, o a creer, de los expertos. Si se entiende que las intuiciones son algún tipo de inclinación a creer, entonces es fácil concluir también que las intuiciones de los expertos son más confiables que las de los legos.

21 En McBain, J.: "Philosophical Intuitions - Philosophical Analisis", en https://mospace.umsystem.edu/xmlui/bitstream/handle/10355/5560/short.pdf? sequence $=2$ (3 de Marzo de 2013). 
y mejor en los temas sobre los que es experto que quien no lo sea. Sus juicios, y sus inclinaciones a realizarlos, por tanto, serán más confiables que aquellos que no hayan pensado tanto y tan detalladamente en estos asuntos. Sus intuiciones, en tanto algún tipo de inclinación a creer entre otras, también serán más confiables. Esto es así, haya o no razones que los avalen. Así, los juicios y las inclinaciones a realizarlos del experto -dentro de las que encontramos sus intuiciones-, serán mejores -más acertadas, con mejor apoyo en la evidencia, más confiables- que las de quien no reflexionó e investigó en el tema.

De todas formas, si se rechaza la idea de la justificación circular, acaso sea posible restituir la vigencia del modelo cartesiano de duda/creencia. ¿Hay algún modo de dirimir la disputa entre cartesianos y no-cartesianos? Una forma de hacerlo parte del siguiente hecho: para zanjar la disputa, hay que apelar a la metodología correcta para dirimir disputas entre posiciones filosóficas. Esa metodología nos insta a fijarnos en cuál de ambas posiciones explica mejor la "base empírica". 22 Dentro de esa "base empírica" va a haber algunas proposiciones a las que estamos fuertemente arraigados. Por ejemplo, que hay un mundo, y que nuestro aparato perceptual es confiable. Desde ya, el cartesiano podría defenderse del no-cartesiano si dice que la disputa no es acerca de la verdad de estas proposiciones, sino acerca de si esas proposiciones están justificadas. El cartesiano, por su parte, si además es escéptico, lo negará, o, al menos, suspenderá el juicio al respecto. En síntesis, el escéptico podrá decir que el debate no es acerca de esas proposiciones, sino acerca de si esas proposiciones están justificadas. Sospecho que dentro de la base empírica en cuestión también están -o deberían estar- las proposiciones de que esas proposiciones de primer orden (hay un mundo, nuestro aparato perceptual es confiable) están justificadas. Pero supongamos que no. ¿Cuál sería la posición escéptica acerca de que hay un mundo, o de que nuestros sentidos son confiables? Si el escéptico lo niega o suspende el juicio al respecto, no está explicando parte de nuestra base empírica, porque ambas proposiciones forman -la primera de modo aún más evidente que la segunda- parte de ella. Si quiere defender que hay un mundo, y que nuestros sentidos son confiables, estará defendiendo que esas proposiciones son verdaderas, pero que no están justificadas -con lo que viola su propio estándar de aceptación de proposiciones, porque acepta proposiciones que no están justificadas-. El no-cartesiano, por su parte, da cuenta de la verdad de todas esas proposiciones.

Una salida disponible para el cartesiano es rechazar la metodología empleada - afirmar que no es la adecuada. Pero esta salida es incorrecta. Entre otras cosas, porque hace del filósofo alguien muy distinto al resto de los investigadores (físicos, matemáticos, economistas o psicólogos), que sí hacen uso de ella. Todos ellos parten de una base empírica a la que explicar, o al menos, a la que recurrir para contrastar teorías. Sostener que esta metodología no funciona para la filosofía, es hacer-

22 Es decir, el conjunto de elementos que sirven para contrastar una teoría. 
la radicalmente distinta a la ciencia. Pero entonces no queda claro en qué sentido es una herramienta para investigar cómo es el mundo -no queda claro en qué sentido la filosofía puede brindar conocimiento-. Lo que le queda, entonces, es rechazar las conclusiones del no-cartesiano, sin rechazar la metodología empleada. Es decir, puede aceptar que la teoría correcta deberá dar cuenta de la base empírica. Solo rechazará que dentro de la base empírica esté la proposición de que hay un mundo, o de que nuestros sentidos sean confiables.

Hay varios modos de interpretar esto. Puede pensarse, por caso, que no hay una base empírica general, sino sólo relativa a individuos. El escéptico cartesiano podría alegar que su base empírica difiere de las de los no-cartesianos. Cierto. Pero el punto relevante debe ser normativo, no meramente descriptivo. Es decir, no alcanza con mostrar individuos o comunidades que acepten (o que lo hagan) que hay un mundo, o que nuestros sentidos son confiables. Lo que sería deseable que el no-cartesiano y anti-escéptico muestre, es que esas proposiciones deberían ser parte de la base empírica. Lo que sería deseable que el cartesiano escéptico muestre, por su parte, es que no deberían serlo.

Así que el cartesiano escéptico no mostró lo que debe mostrar. Pero de lo defendido en este trabajo tampoco se sigue que las proposiciones de que hay un mundo, o que nuestros sentidos son confiables, deben integrar el conjunto de proposiciones aceptadas, y por tanto, la base empírica para formular -al menos algunas- teorías. ${ }^{23}$ Esto es cierto. Es decir, la justificación de ambas proposiciones depende de -i.e., es relativa a- las proposiciones que un individuo dado -o una comunidad dada-hayan aceptado. Si dentro de ese conjunto de proposiciones están las perceptivas -por ejemplo, esto es un teclado, la nieve es blanca y demás-, va a poder justificarse que hay un mundo y que nuestro sistema perceptivo es confiable. Pero si dentro de nuestra base empírica tampoco están estas, no va a haber tal justificación. Es por esto que la presente propuesta, como la dogmática defendida por Pryor, ${ }^{24}$ es modesta. ¿Por qué estas propuestas son modestas? Porque pretenden dar justificación, pero no logran convencer al escéptico. Esto podrá parecer poco, pero nos devuelve algo -la justificación- de lo que el escepticismo cartesiano parecía habernos quitado.

\section{Referencias bibliográficas}

JefFrey, R.: The Logic of Decision. New York: McGraw Hill, 1966.

KLEIN, K.: "Why not Infinitism?", en Cobb-Stevens, R. (ed.), Epistemology: Proceedings of the Twentieth World Congress in Philosophy, 5, 2000, pp. 199-208.

\footnotetext{
23 Acaso ni una ni la otra tengan un papel relevante, por ejemplo, en la justificación de teorías formales.

24 En Pryor, J.: "The Skeptic and the Dogmatist", Nous, 4, 2000, pp. 517-549. Pryor, J.: "What's Wrong with Moore's Argument?", en Sosa, E. y Villanueva, E. (eds.) Philosophical Issues, 14, Epistemology, Atascadero: Blackwell Publishing , 2004, pp. 349-378.
} 
KLEIN, K.: "Infinitism is the Solution to the Regress Problem", en Sosa, E. y Steup, M. (eds.), Contemporary Debates in Epistemology, 2005, pp. 131-139.

LeVI, I.: "On Indeterminate Probabilities", Journal of Philosophy 71: 391-418, 1974.

LeVI, I.: The Enterprise of Knowledge. Cambridge, Mass: The MIT Press, 1980.

MACHERY, E., Mallon, R., Nichols, S. y Stich, S.: "Semantics Cross-Cultural Style", Cognition, 92, B1-B12, 2004.

McBaIn, J.: "Philosophical Intuitions - Philosophical Analysis", en https://mospace.umsystem.edu/xmlui/bitstream/handle/10355/5560/short.pdf?sequence $=2(3$ de Marzo de 2013).

Pryor, J.: "The Skeptic and the Dogmatist", Nous, 4, 2000, pp. 517-549.

PRYOR, J.: "What's Wrong with Moore's Argument?", en Sosa, E. y Villanueva, E. (eds.), Philosophical Issues, 14. Epistemology, Atascadero: Blackwell Publishing, 2004, pp. 349-378.

TURRI, J.: "On the Regress Argument for Infinitism", Synthese 166:1, 2009, pp. 157163.

WeinBerg, J., Nichols, S. y Stich, S.: "Normativity and Epistemic Intuitions" Philosophical Topics, 29: 2001, pp. 429-460.

Williamson, T.: "Philosophical 'Intuitions' and Scepticism about Judgement", Dialectica Vol. 58, No 1, 2004, pp. 109-153.

Williamson, T.: "Philosophical Expertise and the Burden of the Proof", en http://www.philosophy.ox.ac.uk/_data/assets/pdf_file/0013/19300/metaphilosophy.pdf (4 de Enero de 2013).

Federico Matías Pailos

Universidad de Buenos Aires, CONICET

fpailos@hotmail.com 